\title{
Experimental study on Error Modeling and compensation based on non-contact laser coordinate measuring machine
}

\author{
Liu Jia ${ }^{a}$, Zhao $\mathrm{Ji}^{\mathrm{b}}$, Yang $\mathrm{Xu}^{\mathrm{c}}{ }^{*}$, Qu Xingtian ${ }^{\mathrm{d}}$, Wang Xin ${ }^{\mathrm{e}}$ and Liu Jiming ${ }^{\mathrm{f}}$
}

Dept. of Mechanical Manufacturing and Automation, Ji Lin University, Chang Chun 130025, China

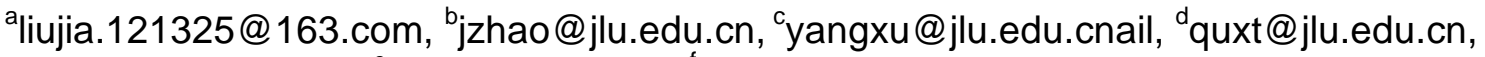
ewxin@jlu.edu.cn, ${ }^{f}$ liujiming3118@163.com

Keywords: Non-contact laser coordinate measuring machine, Error modeling, Error compensation Abstract. In order to improve the measurement accuracy of the non-contact laser coordinate measuring machine, both the error model of coordinate measuring machine and laser measurement are built based on blade measurement. The influence of the surface color and surface inclination angle of the measured object on the measurement errors are studied respectively through the experiment. The error curve shows the practicability and correctness of the error model of laser measurement. And then, the error compensation function is established based on error representation method. By the standard ball test, the measurement accuracy after compensation can reach to micron level and testifies the error compensation model is reasonable.

\section{Introduction}

Coordinate measuring machine as a precision measurement instrument has been development relatively mature in nearly 30 years, the measurement accuracy of contact measuring machine can reach to nano level through error compensation [1]. The coordinate measuring machine with a non-contact laser measuring head becomes a trend to solve the efficiency problem that is the disadvantage of contact measurement $[2,3]$. Wang $\mathrm{G}$ et al proposed the optical model and calibration method of laser triangulation measuring system [4]. Feng $\mathrm{H} \mathrm{Y}$ et al analyzed the digital error of laser scanning system in [5]. Zexiao $\mathrm{X}$ studied the error model and calibration method of laser scanning system in [6]. In this paper, the error modeling and compensation of the coordinate measuring machine with a laser scanner based on the coordinate measuring machine in laboratory is realized whose result prove that the compensation function can improve the accuracy of measurement effectively.

\section{The error model of coordinate measuring machine}
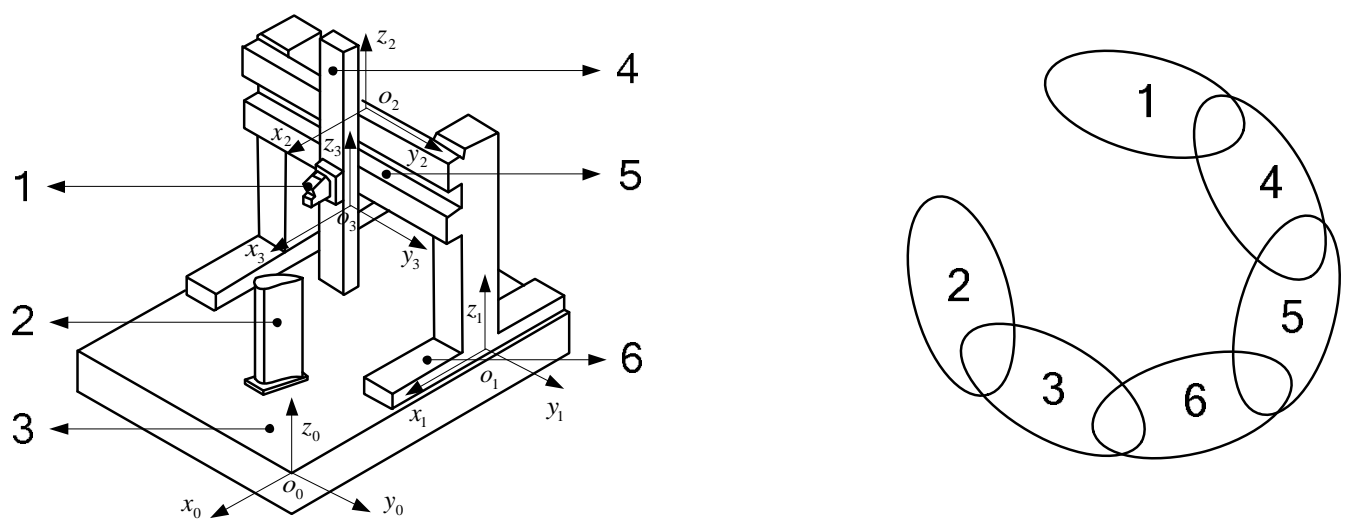

(a) the structure of the coordinate measuring machine (b)the topology structure of the measuring system

1、 laser Measuring head 2、 the measured blade 3、Workbench 4, moving guide along Z direction 5、moving guide along $\mathrm{Y}$ direction 6、 moving guide along $\mathrm{X}$ direction

Fig. 1 the structure of the measuring system

From the Fig. 1, we can see that the position relation between measured blade and laser Measuring head is established through the workbench and moving guides along three directions. According to the 
topology structure of measuring system, the measurement error is obtained by the transmission relationship among the components.

The measurement error of coordinate measuring machine can be calculated by the following formulas.

$$
\begin{aligned}
\Delta x= & \left.\delta_{x}(x)+\delta_{x}(y)+\delta_{x}(z)+z \mid \varepsilon_{y}(x)+\varepsilon_{y}(y)\right]-y_{p}\left[\varepsilon_{z}(x)+\varepsilon_{z}(y)+\varepsilon_{z}(z)\right] \\
& +\mathrm{z}_{p}\left[\varepsilon_{y}(x)+\varepsilon_{y}(y)+\varepsilon_{y}(z)\right]-y \varepsilon_{z}(x)-y \alpha_{x y}-z \alpha_{z x} \\
\Delta y= & \delta_{y}(x)+\delta_{y}(y)+\delta_{y}(z)-z\left[\varepsilon_{y}(x)+\varepsilon_{y}(y)\right]-z_{p}\left[\varepsilon_{x}(x)+\varepsilon_{x}(y)+\varepsilon_{x}(z)\right] \\
& +\mathrm{x}_{p}\left[\varepsilon_{z}(x)+\varepsilon_{z}(y)+\varepsilon_{z}(z)\right]-z \alpha_{y z} \\
\Delta z= & \delta_{z}(x)+\delta_{z}(y)+\delta_{z}(z)-x_{p}\left[\varepsilon_{y}(x)+\delta_{y}(y)+\delta_{y}(z)\right] \\
& +\mathrm{y}_{p}\left[\varepsilon_{x}(x)+\varepsilon_{x}(y)+\varepsilon_{x}(z)\right]+y \varepsilon_{x}(x)
\end{aligned}
$$

where $\delta_{x}(x) 、 \delta_{x}(y) 、 \delta_{x}(z)$ is the linear displacement error of moving guides respectively, $\boldsymbol{\delta}_{y}(x)$ 、 $\delta_{x}(y) 、 \delta_{x}(z) 、 \delta_{z}(x) 、 \delta_{z}(y) 、 \delta_{y}(z)$ is the straightness error of moving guides respectively, $\varepsilon_{x}(x) 、 \varepsilon_{y}(y) 、 \varepsilon_{z}(z)$ is the rotation angle error of moving guides respectively, $\varepsilon_{y}(x) 、 \varepsilon_{x}(y)$ 、 $\varepsilon_{x}(z)$ is the pitch angle error of moving guides respectively, $\varepsilon_{z}(x) 、 \varepsilon_{z}(y) 、 \varepsilon_{y}(z)$ is the bias angle error of moving guides respectively, $\alpha_{x y}, \alpha_{y z}, \alpha_{x z}$ is the error of perpendicularity of moving guides respectively, $\delta_{i}(j)$ indicates that the linear displacement error and straightness error are derived from the axial displacement of $j$ axis on moving guide of $i$ axis, $\varepsilon_{i}(j)$ indicates that the angle error is derived from the axial displacement of $j$ axis on moving guide of $i$ axis, $\alpha_{i j}$ indicates that the error of perpendicularity between axes $j$ and $i$.

\section{The error model of Laser measurement}

When the beam of laser displacement sensor is projected onto the surface of the measured object, the modulation effect will be generated on the surface of measured object, and the space scattered field is formed after the light scattering. The light spot is obtained by receiving the reflected light on the PSD photosensitive surface based on the photoelectric detector. The position of the light spot determines the change of the optical output of PSD and the surface distance of measured object. The not uniform intensity distribution of the light spot, the size of the spot diameter and the inclination angle of the measured object surface can lead to the change of the position of light spot, and cause the error of the measurement.

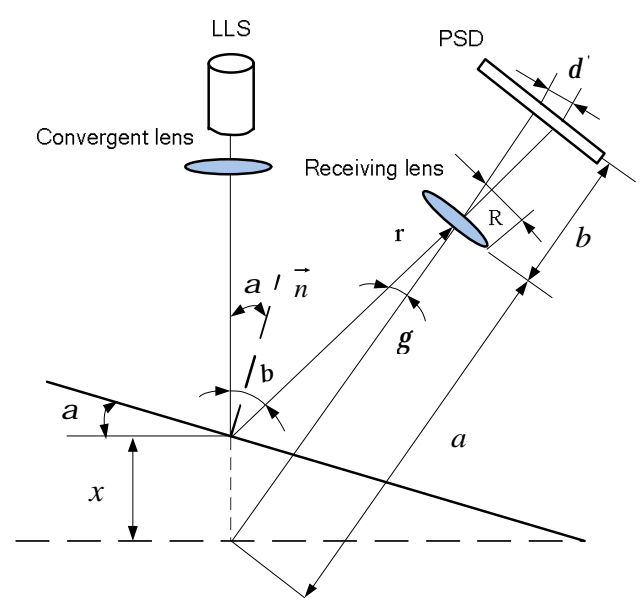

Fig. 2 Laser measurement principle 
When the surface of the measured object has an angle of inclination with the horizontal plane, the laser measurement principle is shown in Fig. 2. As shown in Fig. 2, $a, b$ are the structure parameter, $x$ is the distance variation of measured object surface, The inclination angle $\alpha$ is positive when the beam rotates counterclockwise with respect to normal, $R$ is the effective light radius of the receiving lens, $r$ is the distance between the light point of the measured object's surface and the center of the receiving lens is measured, $\gamma$ is the angle between the reflected light and the optical axis of the receiving lens, $\delta$ is the displacement deviation of the light spot on the PSD surface of the light detector.

According to the geometric relations shown in Fig. 2, the measurement error of the surface distance of the measured object which caused by the deviation of the light center position on the PSD surface of the light detector is:

$$
\Delta x=x \frac{R^{2}}{a^{2}}\left(1+2 \frac{x}{a} \cos \beta\right)[\tan \beta-\tan (\beta-\alpha)]
$$

\section{Experiment}

The error calibration of the coordinate measuring machine can be referred to [7]. In this paper, we mainly study the error compensation of laser measurement.

1. Effect of measured objects' surface with an angle of inclination on the nonlinear error of the measuring system

The laser measuring head is mounted on the moving guide along $\mathrm{Z}$ direction, parallel to the workbench which ensures the incident beam parallel with digital rotary platform. In the measurement process, the $\mathrm{X}$ and $\mathrm{Y}$ axes remain stationary, and the $\mathrm{Z}$ axis works. The $80 \mathrm{~mm}$ gauge vertically placed and fixed on the digital rotary platform whose working plane parallel to the YZ plane of coordinate measuring machine. Set the output value of relative distance of laser displacement sensor is zero which is regarded as the measurement reference point. Moving the $\mathrm{Z}$ axis with the step size of $0.5 \mathrm{~mm}$, moving 5 steps to measure 5 data points and each point of 5 times. And then, the 25 data is recorded and calculated the average error as the error value under this condition. Since then, the rotation platform is rotated $10^{\circ}$ along counterclockwise in once time, and the error value of this condition is calibrated. In the counterclockwise case, we obtain 6 sets of error values in different angles respectively. In the clockwise case, we obtain 6 sets of error values in the same way.

We obtained 65 measured points in 13 groups of different angles. Different curves represent the error values of different moving distance as shown in Fig. 3. From the Fig. 3 we can see that the measurement error is increased with the increase of the inclination angle of the surface of the measured object and they have the positive correlation. When the incident beam is parallel to the normal direction of the measured surface, the measurement error caused by the inclination angle can be negligible. And the measurement error curve is axis symmetry which is reasonable. 


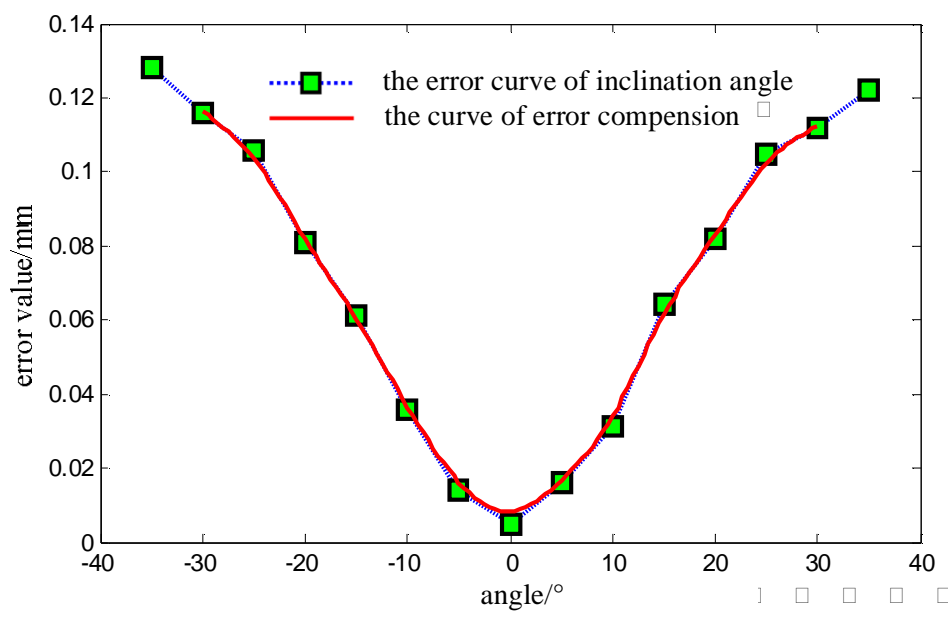

Fig. 3 the measurement error of different inclination angle

2. Effect of measured objects' surface color on the error of the measuring system

The parameters of laser displacement sensor are determined by the object of the test which is a piece of white paper. However, the actual measured workpieces often are metal parts. It is necessary to study the influence of different surface color on the error. The $20 \mathrm{~mm}$ gauge is placed on the rotary platform and the long side of the $20 \mathrm{~mm}$ gauge working plane is parallel to the $\mathrm{Y}$ axis. We label different color paper on the gauge working plane respectively, and move gauge with the step size of $0.5 \mathrm{~mm}$, moving 10 steps to measure 10 data points and each point of 5 times. And then, the 50 data is recorded and calculated the average error as the error value under this condition. Repeat the above steps until the different colors of paper are all measured.

We obtained 80 measured points with 8 colors of papers. Different curves represent the error values of different colors shown in Fig. 4. From the Fig. 4 we can see that the measured object surface with black have the maximum measurement error. This is because the black surface absorb the light at the highest level which reduce the light energy of receiving lens and cause the great changes of photoelectric output consequently. Similar color has a similar error distribution which shows that the experimental results are reasonable. The experimental results also provide the basis for the surface color processing of the workpieces.
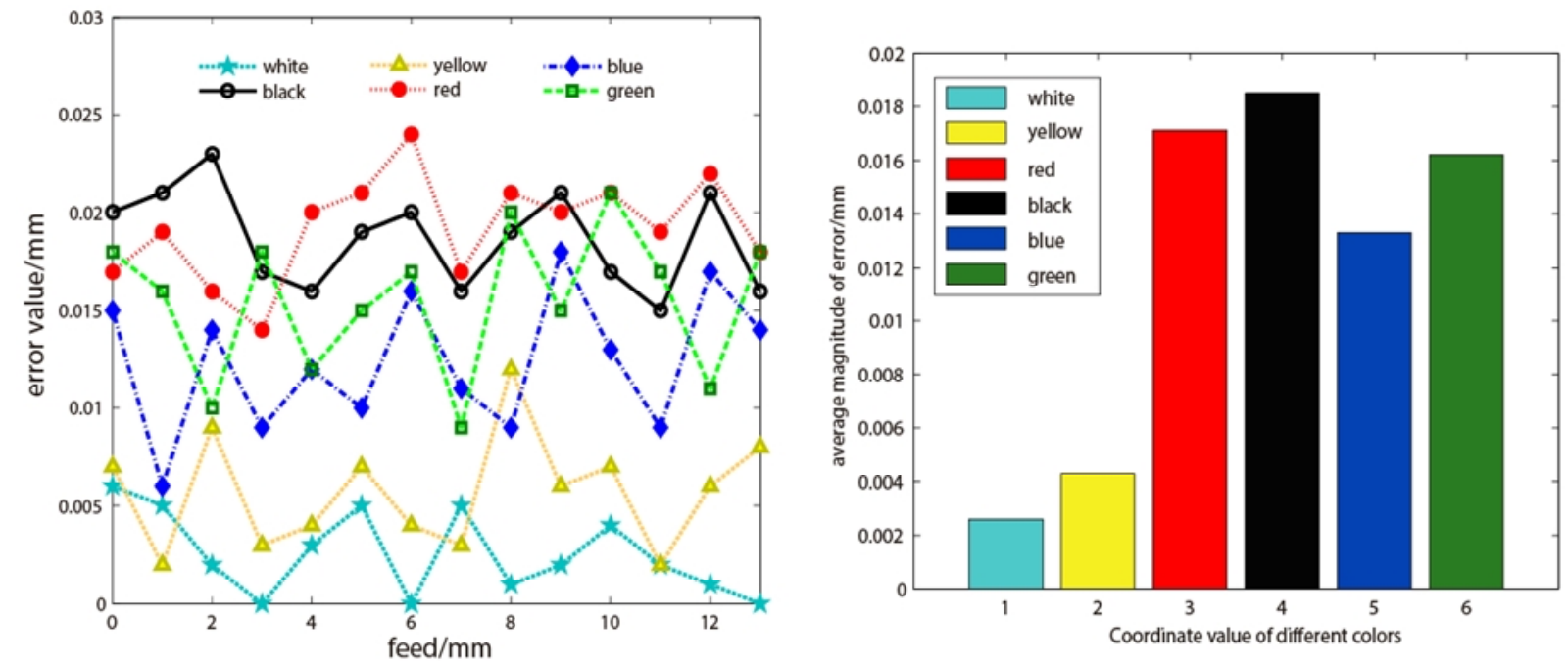

Fig. 4 the measurement error of different surface color

3. Research on the error compensation of non-contact measuring system

The distribution of the compensation error function obtained by the error representation method is used to compensate and correct the measured data. The standard ball test data is used to verify the effectiveness of the compensation error function.

As shown in Fig. 5, we set the measuring step is $1 \mathrm{~mm}$, the scanning range of the standard ball is $10 \times 10 \mathrm{~mm}$, and the inclination angle of the measured point is $-28.6^{\circ} \sim+28.6^{\circ}$. Under this condition, 
100 measured data points is obtained on the standard ball surface. The diameter of the standard ball is $25.4006 \mathrm{~mm}$, that is, the radius is $12.7003 \mathrm{~mm}$. The fitting surface and the fitting surface after the error compensation are shown in Fig. 6.

From the Fig. 6, we can see that the fitting sphere radius of the measured points is $12.6542 \mathrm{~mm}$ and the deviation of radius is $0.0461 \mathrm{~mm}$. The limit deviation of the measured points which is the deviation of the maximum distance between the centre of fitting sphere and measured point and the minimum distance between the centre of fitting sphere and measured point is $0.2377 \mathrm{~mm}$. When the measured points are compensated by using the compensation function, the fitting sphere radius of the measured points is $12.6883 \mathrm{~mm}$ and the deviation of radius is $0.0119 \mathrm{~mm}$. The limit deviation of the measured points which is the deviation of the maximum distance between the centre of fitting sphere and measured point and the minimum distance between the centre of fitting sphere and measured point is $0.2370 \mathrm{~mm}$. Experimental results show that the measurement accuracy after compensation is $0.0009 \mathrm{~mm}$ and is improved by $300 \%$ compared to no compensation.

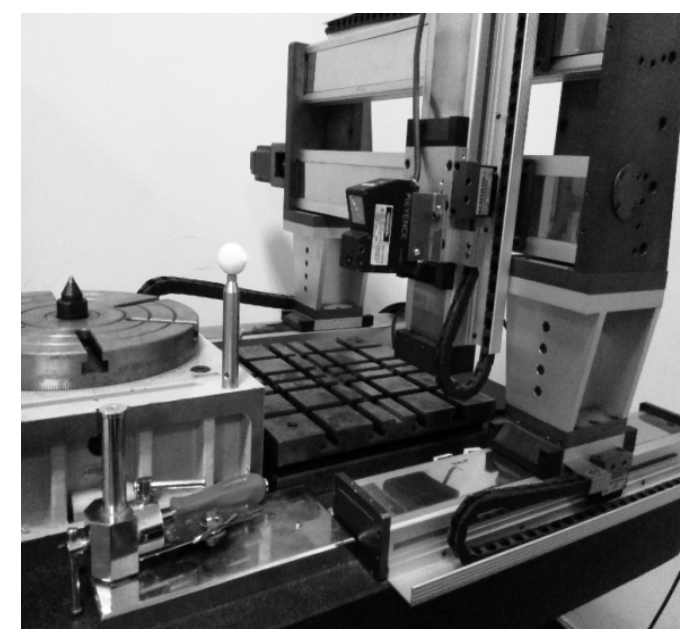

Fig. 5 The measuring experiment of standard ball

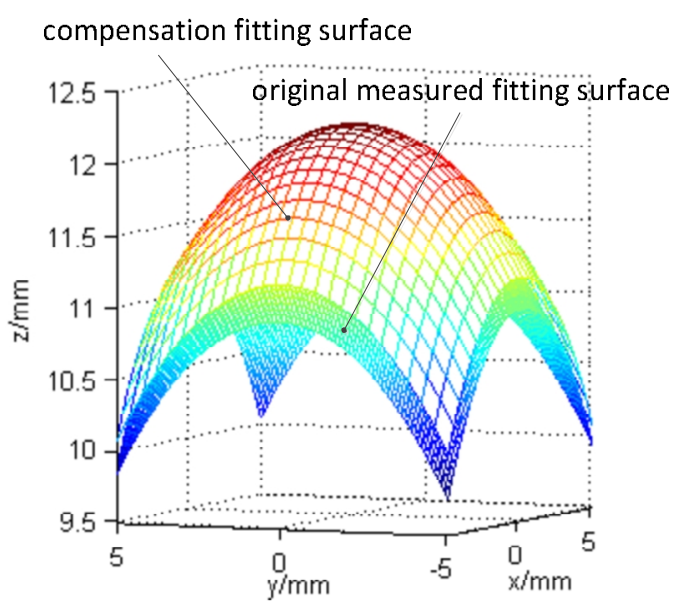

Fig. 6 The fitting surface of the standard ball

\section{Summary}

This paper presents a standard ball test for compensating the measurement error of non-contact coordinate measuring machine. The error models of non-contact coordinate measuring machine and Laser measurement are established according to the theory of multi-body system and Lambert's law respectively. The error of Laser measurement is detected by measuring the object's surface with the different color and inclination angle. The measurement accuracy after error compensation can reach to micron level, which verifies the applicability of the error model.

\section{Acknowledgements}

The authors would like to be grateful to the Key Program of National Natural Science Foundation of China (Grant No. 51135006).

\section{References}

[1] Al-Ahmari A M A, Aalam J. Optimizing parameters of freeform surface reconstruction using CMM[J]. Measurement, 2015, 64: 17-28.

[2] Tasic T, Acko B. Integration of a laser interferometer and a CMM into a measurement system for measuring internal dimensions[J]. Measurement, 2011, 44(2): 426-433. 
[3] Gąska A, Sładek J, Ostrowska K, et al. Analysis of changes in coordinate measuring machines accuracy made by different nodes density in geometrical errors correction matrix[J]. Measurement, 2015.

[4] Wang G, Zheng B, Li X, et al. Modelling and calibration of the laser beam-scanning triangulation measurement system[J]. Robotics and Autonomous Systems, 2002, 40(4): 267-277.

[5] Feng H Y, Liu Y, Xi F. Analysis of digitizing errors of a laser scanning system[J]. Precision Engineering, 2001, 25(3): 185-191.

[6] Zexiao X, Chengguo Z, Qiumei Z. A simplified method for the extrinsic calibration of structured-light sensors using a single-ball target[J]. International Journal of Machine Tools and Manufacture, 2004, 44(11): 1197-1203.

[7] Sun Q Z, Zhao J, Ji S J, et al. Error Detection Technology for Calibrating Precision of Non-Contact Coordinate Measuring Machine[C]//Key Engineering Materials. 2014, 625: 42-46. 